\title{
Correction to: Prescribing Patterns and Impact of Factors Associated with Time to Initial Biologic Therapy among Children with Non-systemic Juvenile Idiopathic Arthritis
}

\author{
Xiaomeng Yue 1,6 (Din Huang ${ }^{2,3} \cdot$ Ana L. Hincapie ${ }^{1} \cdot$ Patricia R. Wigle ${ }^{1} \cdot$ Tingting Qiu $^{2} \cdot$ Yuxiang $\mathrm{Li}^{4}$. \\ Esi M. Morgan ${ }^{3,4,5}$. Jef J. Guo'
}

Published online: 21 April 2021

(C) The Author(s), under exclusive licence to Springer Nature Switzerland AG part of Springer Nature 2021

\section{Correction to: Pediatric Drugs (2021) 23(2):171-182 https://doi.org/10.1007/s40272-021-00436-4}

Unfortunately, the original article has been published with the incomplete author affiliations.

Page 182: The listing of the author names and affiliations, which previously read:

Xiaomeng Yue ${ }^{1} \cdot$ Bin Huang $^{2,3} \cdot$ Ana L. Hincapie ${ }^{1} \cdot$ Patricia R. Wigle ${ }^{1}$. Tingting $\mathrm{Qiu}^{2} \cdot$ Yuxiang $\mathrm{Li}^{4} \cdot$ Esi M. Morgan ${ }^{3,4,5}$ - Jef J. Guo ${ }^{1}$

${ }^{1}$ Division of Pharmacy Practice and Administrative Sciences, James L. Winkle College of Pharmacy, University of Cincinnati, 3225 Eden Ave., Cincinnati, OH 45267, USA

${ }^{2}$ Division of Biostatistics and Epidemiology, Cincinnati Children's Hospital Medical Center, Cincinnati, OH, USA

The original article can be found online at https://doi.org/10.1007/ s40272-021-00436-4.

Xiaomeng Yue

yuexn@mail.uc.edu

1 Division of Pharmacy Practice and Administrative Sciences, James L. Winkle College of Pharmacy, University of Cincinnati, 3225 Eden Ave., Cincinnati, OH 45267, USA

2 Division of Biostatistics and Epidemiology, Cincinnati Children's Hospital Medical Center, Cincinnati, OH, USA

3 Department of Pediatrics, University of Cincinnati College of Medicine, Cincinnati, OH, USA

4 Department of Environmental and Public Health Sciences, University of Cincinnati College of Medicine, Cincinnati, $\mathrm{OH}, \mathrm{USA}$

5 Division of Rheumatology, Cincinnati Children's Hospital Medical Center, Cincinnati, OH, USA

6 Present Address: AbbVie Inc., North Chicago, IL, USA
${ }^{3}$ Department of Pediatrics, University of Cincinnati College of Medicine, Cincinnati, OH, USA

${ }^{4}$ Department of Environmental and Public Health Sciences, University of Cincinnati College of Medicine, Cincinnati, OH, USA

${ }^{5}$ Division of Rheumatology, Cincinnati Children's Hospital Medical Center, Cincinnati, OH, USA

Should read:

Xiaomeng Yue ${ }^{1,6} \cdot$ Bin Huang $^{2,3} \cdot$ Ana L. Hincapie ${ }^{1} \cdot$ Patricia R. Wigle ${ }^{1} \cdot$ Tingting $\mathrm{Qiu}^{2} \cdot$ Yuxiang $\mathrm{Li}^{4} \cdot$ Esi M. Morgan ${ }^{3,4,5}$ - Jef J. Guo ${ }^{1}$

${ }^{1}$ Division of Pharmacy Practice and Administrative Sciences, James L. Winkle College of Pharmacy, University of Cincinnati, 3225 Eden Ave., Cincinnati, OH 45267, USA

${ }^{2}$ Division of Biostatistics and Epidemiology, Cincinnati Children's Hospital Medical Center, Cincinnati, OH, USA

${ }^{3}$ Department of Pediatrics, University of Cincinnati College of Medicine, Cincinnati, OH, USA

${ }^{4}$ Department of Environmental and Public Health Sciences, University of Cincinnati College of Medicine, Cincinnati, OH, USA

${ }^{5}$ Division of Rheumatology, Cincinnati Children's Hospital Medical Center, Cincinnati, OH, USA

${ }^{6}$ Current affiliation: AbbVie Inc., North Chicago, IL, USA 